\title{
Article
}

\section{Framing and visual type: Effect on future Zika vaccine uptake intent}

\author{
Jeanine P.D. Guidry, ${ }^{1}$ Kellie E. Carlyle, ${ }^{2}$ Jessica G. LaRose, ${ }^{2}$ Paul Perrin, ${ }^{3}$ Mark Ryan, ${ }^{4}$ \\ Marcus Messner, ${ }^{1}$ Jay Adams ${ }^{1}$ \\ ${ }^{1}$ Robertson School of Media and Culture, ${ }^{2}$ Department of Health Behavior and Policy, ${ }^{3}$ Department of \\ Psychology, ${ }^{4}$ Department of Family Medicine, Virginia Commonwealth University, Richmond, VA, USA
}

\begin{abstract}
Significance for public health
The study described in this paper is significant for the field of public health for several reasons: It takes a proactive approach in studying messaging focused on the Zika vaccine before that vaccine is available, allowing for quick implementation of its limited results. In addition, this study centers on messaging in the form of realistic images consistent with those that could be posted on Instagram, thereby focusing on a relatively new yet immensely popular communications platform that few are focusing on presently.
\end{abstract}

\begin{abstract}
Introduction: The Zika virus is associated with the birth defect microcephaly, and while a vaccine was not available in early2017, several were under development. This study's purpose was to identify effective communication strategies to promote uptake of a new vaccine, particularly among women of reproductive age.

Design and methods: In order to study the effects of Zika message framing (gain $v s$. loss) and visual type (photo $v s$. infographic) on future Zika vaccine uptake intent, a $2 \times 2$ between-subjects experiment was performed via an online survey in 2017 among 339 U.S. women of reproductive age (18-49 years). Participants were exposed to one of four messages, all resembling Instagram posts: gain-framed vs. loss-framed infographic, and gain-framed $v s$. loss-framed photo. These messages were followed by questions about Zika vaccine uptake intent as well as intermediate psychosocial variables that could lead to intent.

Results: There was no interaction between framing and visual type $(\mathrm{P}=0.116)$, and there was no effect for framing $(\mathrm{P}=0.185)$ or visual type $(\mathrm{P}=0.724)$ on future $\mathrm{Zika}$ vaccine uptake intent, which is likely indicative of insufficient dosage of the intervention. However, when focusing on intermediate psychosocial constructs that are known to influence behavior and intent, gain-framed messages were more effective in increasing subjective norms $(\mathrm{P}=0.005)$ as related to a future Zika vaccine, as well as perceived benefits $(\mathrm{P}=0.016)$ and self-efficacy $(\mathrm{P}=0.032)$.

Conclusions: Gain-framed messages seem to be more effective than loss-framed messages to increase several constructs that could, in turn, affect future Zika vaccine uptake intent. This is a novel finding since, traditionally, loss-framed messages are considered more beneficial in promoting vaccine-related health behaviors.
\end{abstract}

\section{Introduction}

Until recently, Zika was a relatively unknown virus with mild flu-like symptoms. However, in the past few years it has quickly transformed into a global health threat across 84 countries, territories, or subnational areas with evidence of Zika transmission. ${ }^{1}$ Originally thought to be spread only by mosquitoes, we now know the virus can also be transmitted sexually, via blood transfusions, and from mother to fetus during pregnancy. The severity of Zika is perhaps best illustrated by one of its most devastating consequences: a study by the New England Journal of Medicine estimated that between $1-13 \%$ of women who contract Zika while pregnant could give birth to a baby with microcephaly, an underdeveloped brain frequently accompanied by other abnormalities. ${ }^{2}$ No vaccine is currently available, but several vaccine candidates are under development and in clinical trial testing phases.

Public health emergencies such as infectious disease outbreaks like Zika require quick, effective communication about both the issue itself and the availability of interventions. ${ }^{3}$ While social media can play a significant role in distributing reliable information, it can just as easily contribute to the viral spread of misinformation, such as the increasing presence of vaccine-hesitant posts online. Therefore, it is critical to develop future Zika vaccine messaging and communication strategies specifically for social media communications. ${ }^{4}$ While few Zika vaccine messages were present on social media platforms since the vaccine is not available to the public yet, several studies have looked at online Zika virus conversations. A study focused on Google searches, tweets, and Associated Press wire stories found that news coverage of Zika-related public health authority announcements opens brief windows of information sharing, engagement, and searching, which can be used for education purposes. ${ }^{5}$

Among social media platforms, the mobile social networking platform Instagram is of particular interest. The photo- and videosharing platform is primarily a mobile application and has become one of the main visual engagement channels for smartphones and tablets. Instagram's growth has been rapid, surpassing 700 million users as of April 2017. ${ }^{6}$ The Zika epidemic is international in nature, and one of Instagram's strengths is its ability to communicate across languages with visuals. In addition, a study of Zikafocused Instagram messages found that almost all images with a target audience focused on women, and that half of the images expressed negative sentiments such as fear. ${ }^{7}$ As such, Instagram is the medium of focus in this study.

Specific predictors both message-related as well as psychosocial - for future Zika vaccine uptake are not known. However, predictors of vaccine uptake are known for other pandemic vaccines like H1N1, as well as for vaccines like the HPV vaccine, and these might provide insight into future Zika vaccine predictors and aid in future communications planning. This paper examines two key message-related variables - message framing and visual type - and their influence on reported future Zika vaccine uptake intent, as well as on the psychosocial variables leading to intent as described by leading health behavior change theories. Although 
the specifics of these psychosocial variables may change as the vaccine becomes available, health decisions are constantly impacted by the evolving social environment and public health exigencies, such as Zika, require proactive approaches by health communication professionals.

\section{Message-related variables}

\section{Gain and loss framing}

Message framing can have an impact on the ultimate effect of messages on health behavior intent and actions. One of the most often used framing techniques is gain- versus loss-framed messages. Gain-framed messages generally focus on the benefits of engaging in a specific behavior, while loss-framed messages focus on the consequences of not engaging in a particular behaviour. 8,9

Studies show that gain-framed messages tend to be more effective in promoting greater adherence to prevention behaviors, such as physical activity. ${ }^{8}$ These preventive behaviors are perceived as safe and less risky because they serve to prevent a future health issue. ${ }^{9}$ Loss-framed messages are more effective in promoting illness-detecting, diagnostic behaviors such as HIV screenings. ${ }^{10}$ Diagnostic behaviors are perceived as risky or uncertain because people may find out they have a potentially serious illness. ${ }^{9}$

Given the extant literature, it would be reasonable to hypothesize that vaccinations, a known preventive behavior, would be most effectively promoted using gain-framed messages. However, several studies have found that, instead, loss-framed messages work better to promote the HPV vaccine, ${ }^{11}$ the MMR vaccine, ${ }^{12}$ and the H1N1vaccine. ${ }^{13}$ A reason for this may be that, while vaccinations are preventive behaviors, they are also often associated with presumed, often disproven adverse consequences. While there are several studies available on the representation of different vaccines on social media platforms - showing that online conversations about vaccines contain a mix of pro- and anti-vaccine messages $^{14-16}$ - so far no studies have focused on testing the use of gain- and loss-framing in online vaccine messaging. There is both a gap in knowledge regarding how these dynamics may function in online, visual, social environments as well as a more general gap in the understanding of the inconsistent findings in gain/loss messaging effects in the vaccine context. These are two of the gaps in knowledge this study endeavored to address.

\section{Types of visual information}

The concept of risk is often a difficult one for people to grasp. ${ }^{17}$ Most risk information is either portrayed as numbers alone or as a combination of numbers and text, but visual representations can facilitate comprehension and recall of risk information. ${ }^{17}$ Visuals of all kinds - including graphs, infographics, and photos are recommended and used more frequently than numeric and verbal communications of risk. ${ }^{17}$ Graphics-visual displays such as histograms, pie charts, stick figures, dots, and line charts, can improve the comprehension of numeric risk, revealing patterns that are not easily visible otherwise and attracting attention by displaying information in concrete terms. ${ }^{18}$

It is well established that visuals are processed differently than text-based messages: Dual coding theory, for example, explains that visuals have an advantage over text because they are coded into both visual as well as verbal memory and are more easily retrieved from the brain because they are encoded more uniquely. ${ }^{19-21}$ Information communicated through visuals, and text accompanied by visuals, increase attention to and recall of health education information compared to text alone. This may be of particular significance when communicating with those with lower literacy - often from vulnerable populations - who may not possess the literacy skills to read, interpret, and act on text-only health information. ${ }^{19}$ Visuals often serve to improve risk comprehension as well as the processing of other types of complex information. ${ }^{17}$ Finally, human brains process visual images with great speed and respond to them substantially faster than to verbal symbols, which is particularly relevant considering the average time spent on social media messages. ${ }^{22}$

The next, relatively unanswered, question, however, is whether there is a difference between distinct types of visuals and their effect on message comprehension and risk perception. Infographics - graphic representations of information - are a popular tool for presenting online health information. ${ }^{23}$ Infographics boost understanding of health information by utilizing a person's visual ability to see patterns and trends, can present complex information or data in a visual format that is both quick and easy to comprehend, and can increase cognitive functions such as inference making. ${ }^{24}$ Regular photographs overlaid with text - whether in color or black and white - are an example of what Houts et al. call a combination of visual and text. ${ }^{19}$ These types of visuals have not been the focus of much research, but as far as format, seem to be similar to Internet memes, which tend to have a high level of virality. 25

\section{Research questions and hypothesis}

The research reviewed thus far makes it clear that visual type and framing are important message characteristics to consider. However, to date these have not been studied in conjunction, therefore we begin with a research question that explores this interaction: $R Q 1$ : Do message frame and visual type interact to influence intent to receive the Zika vaccine?

There is more evidence regarding gain- and loss-frames as they relate to vaccine uptake. As stated earlier, studies show that in many cases, loss-framed messages are more likely than gainframed messages to promote vaccine uptake. ${ }^{13,26,27}$ Thus, this study's hypothesis is:

H1: Messages with a loss frame will be more likely to result in women reporting intent to get the Zika vaccine than messages with a gain frame.

Conversely, while there are reasons to think infographics and photos with text may have a different effect, there is not enough evidence yet to know with confidence. The second research question, therefore, is:

RQ2: Are there differences in intent to get the Zika vaccine between women who receive the infographic $v s$. the photo/text message?

Having narrowed the focus to message frame and type of visual, this paper now turns to the role of health behavior theory in designing message content.

\section{Health behavior theories}

Health behavior theories are an essential component of designing effective public health messaging. Two of the most frequentlyused theories are the Health Belief Model (HBM) and the Theory of Planned Behavior (TPB). ${ }^{28,29}$ The HBM's constructs are perceived severity, perceived susceptibility, perceived benefits, perceived barriers, self-efficacy, and cues to action. The TPB's constructs are attitudes, subjective norms, perceived behavioral control, and behavioral intent.

The HBM was developed specifically for preventive behaviors such as vaccination; however, examining vaccination behavior through the lens of both the HBM and TPB offers several advan- 
tages. First, the prevalence of social media has brought with it an increased focus on the normative components of health behavior theories. This is an advantage of including the TPB, as subjective norm is one of its three main constructs. Additionally, as with all behaviors, there is a difference between vaccine uptake and vaccine uptake intent. For this study, only vaccine update intent is used because, while the Zika vaccine is under development, it is not available yet. The TPB explicitly distinguishes between intent and behavior, making it particularly appropriate for current Zika vaccine application.

\section{Health belief model}

Higher seasonal flu vaccine uptake is associated with low perceived barriers to obtaining the vaccine, high perceived susceptibility to contracting the flu, high perceived benefits of the vaccine, high perceived severity of the flu and high self-efficacy for obtaining the vaccine, and cues to action. ${ }^{30}$ Higher H1N1 vaccine uptake is also associated with low perceived barriers. ${ }^{31}$ Similarly, higher seasonal flu vaccine uptake intent is associated with high perceived susceptibility to the flu, along with high perceived benefits of and low perceived barriers to getting the vaccine and cues to action. ${ }^{32}$ Higher H1N1 flu vaccine uptake intent is associated with high susceptibility to the H1N1 flu, high perceived severity, high perceived benefits of the $\mathrm{H} 1 \mathrm{~N} 1$ vaccine, and cues to action. ${ }^{33}$

\section{Theory of planned behavior}

Lower seasonal flu vaccine uptake is associated with higher levels of negative attitudes to the vaccine. ${ }^{31}$ Higher H1N1 vaccine uptake is associated with higher positive subjective norm, ${ }^{27}$ while lower H1N1 flu vaccine uptake, similar to the seasonal flu vaccine, is associated with higher negative attitudes toward to getting the vaccine. ${ }^{31}$ Higher HPV vaccine uptake, meanwhile, is associated with higher positive subjective norms. ${ }^{34}$ Moreover, higher H1N1 flu vaccine uptake intent is associated with a positive attitude toward the H1N1 vaccine, positive subjective norms, and a higher level of perceived behavioral control related to vaccine uptake intent. ${ }^{33}$ Higher HPV vaccine uptake intent, meanwhile, is associated with a positive attitude toward the HPV vaccine, and with positive subjective norms related to the vaccine. ${ }^{35}$ The third research question of this study is, therefore:

RQ3: What message characteristics are most effective at increasing

the intermediate psychosocial constructs predicted by the HBM and TPB associated with intent to get a future Zika vaccine?

\section{Design and Methods}

The psychosocial and behavioral impacts of Zika vaccine message framing and image type were examined via a $2 \times 2$ betweensubjects experiment with a U.S. sample of 339 women of reproductive age in March 2017. Participants were first provided with the following text: The Zika virus (Zika) can be spread through mosquitos, through sexual transmission, and from a pregnant woman to her fetus. Most of the symptoms of Zika are mild, but the Zika infection during pregnancy can cause fetuses to have a serious birth defect of the brain called microcephaly - a medical condition in which the brain does not develop normally. Currently, no vaccine or treatment is available for Zika, but several versions of a vaccine are under development and could be available as early as sometime in 2017, and were then randomized to one of four arms: gain-framed photo/text $(n=78)$, gain-framed infographic $(n=90)$, loss-framed photo/text $(n=83)$, or loss-framed infographic $(n=88)$ (Figure 1), followed by a questionnaire focused on attitudes and beliefs related to the future Zika vaccine, and with the main outcome being intent to get the vaccine. All procedures were approved by the Institutional Review Board (IRB) at a large research university in the Mid-Atlantic U.S. The experiment was conducted online, with participants exposed first to the stimuli message for their respective condition and then asked to complete a survey questionnaire.

\section{Stimuli development}

Four versions of the stimuli message about the future Zika vaccine were created in the visual form of an Instagram post (Figure 1), incorporating the following independent variables: message framing (gain vs. loss) and visual type (photo vs. infographic). To maximize internal validity, the Zika virus information and Zika vaccine recommendation were held constant across conditions, as were color and formatting. The stimuli were reviewed by a panel of experts in message design using an iterative process until they reached agreement that the manipulations of frame and visual type had strong face validity and the remaining message content was as consistent as plausible for the design. External validity was addressed by creating stimuli that appeared as if they were screenshots from Instagram posts originating with the Centers for Disease Control and Prevention (CDC), because it has established itself as the online public health authority with respect to infectious disease outbreaks like the H1N1 outbreak and the recent Ebola epidemic. ${ }^{36,37}$

\section{Recruitment procedures}

A nationwide sample of 339 participants was recruited by Qualtrics, a survey research firm. Quota sampling by U.S. geographic region was used to help achieve a geographically balanced sample. Data collection was initiated and completed in March 2017.

\section{Measures}

Measures included demographic variables (age, region, ethnicity, education, and income) and healthcare-related variables (previous seasonal flu vaccine uptake; future seasonal vaccine uptake intent; and having a regular healthcare provider).

HBM and TPB constructs were assessed using scales adapted from Myers and Goodwin. ${ }^{33}$ All measures showed good reliability

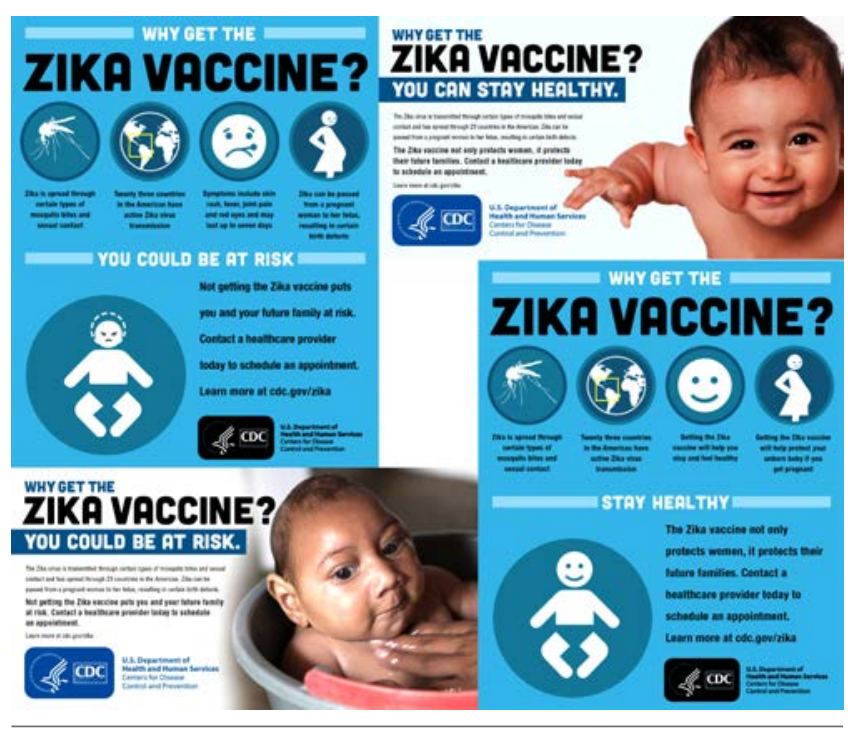

Figure 1. Loss-framed infographic (top left), loss-framed photo (bottom left), gain-framed photo (top right), and gain-framed infographic (bottom right). 
(ranging from 0.75 to 0.97 ), and were measured on a six-point Likert scale, ranging from strongly disagree to strongly agree unless otherwise noted. These measures included perceived severity and perceived susceptibility of the Zika virus (both measured on a seven-item Likert scale), perceived benefits of and barriers to the vaccine, self-efficacy, and cues to action (response options yes and no). Attitude was measured using five semantic differential responses. In addition, subjective norms and perceived behavioral control were measured. Finally, respondents' intentions to get the Zika vaccine when it becomes available were measured using a single item.

\section{Analytic approach}

Simmons, Nelson, and Simonsohn state that cells in statistical analyses should include at least 20 observations. ${ }^{38}$ Therefore, the lowest two education variable options, some high school and high school completed, were combined into one high school or less option. In addition, only Caucasian, African-American, and Hispanic ethnicity options were included in the analyses due to this constraint. Descriptive analyses were conducted for the stimulus manipulation variables of gain/loss framing and photo/infographic visual type, as well as for vaccine intent. A two-way analysis of variance (ANOVA) was used to test the following effects on the primary dependent variable, vaccine uptake intent, as expressed by a six-level Likert outcome (and regarded as a continuous variable): the interaction between framing and visual type, main effect of framing, and main effect of visual type, as appropriate. Subsequently, two-way ANOVAs were used to test the interaction between framing and visual type as well as main effects of framing and visual type (where appropriate) on intermediate psychosocial constructs: Attitudes, subjective norms, perceived behavioral control, perceived severity and susceptibility, perceived benefits and barriers, self-efficacy, and cues to action. Lastly, psychosocial construct items were examined individually to gain a more nuanced understanding of the observed relationships.

\section{Results}

\section{Participant characteristics}

The mean age of the respondents was 33.9 ( $\mathrm{SD}=7.88$ ). Most participants were from the South (38.9\%), followed by the Western region $(24.5 \%)$, Midwest $(20.9 \%)$, and Northeast $(15.6 \%)$. In terms of education, $4.1 \%(n=14)$ reported having some high school, 20.4\% $(n=69)$ a high school diploma, 33.0\% $(n=112)$ some college, $11.5 \%(n=39)$ reported getting a 2-year degree, $22.4 \%$ $(n=76)$ a 4-year college degree, and $8.6 \%(n=29)$ reported having a graduate degree. Finally, 9.4\% $(\mathrm{n}=32)$ were African-American, $1.2 \%(n=4)$ American Indian, 5.0\% $(n=17)$ Asian, 8.8\% $(n=30)$ Hispanic, $73.5 \%(n=249)$ Caucasian, and $2.1 \%(n=7)$ other.

When asked for their response to the question I intend to get the future Zika vaccine when it becomes available, $25.4 \%(\mathrm{n}=86)$ indicated strongly agree, $26.5 \%(\mathrm{n}=90)$ agree, and $25.1 \%(\mathrm{n}=85)$ somewhat agree. In addition, $6.8 \%(\mathrm{n}=23)$ responded strongly disagree, $8.6 \%(\mathrm{n}=29)$ disagree, and $7.7 \%(\mathrm{n}=26)$ somewhat disagree. Collapsing this, $77.0 \%(\mathrm{n}=261)$ of the study respondents reported they agreed to an extent with getting the Zika vaccine, while $23.0 \%$ $(\mathrm{n}=78)$ disagreed to an extent $(\mathrm{M}=4.3, \mathrm{SD}=1.49)$.

A two-way ANOVA was conducted to examine the effects of framing and visual type on intent to get the future Zika vaccine. Data were normally distributed, as assessed by a Q-Q plot. There were six outliers, as assessed by standardized scores greater than
3.0. These outliers were left in the analysis, since Cohen suggests leaving in a limited number of outliers $(1-2 \%$, in this case just under $2 \%$ ) that are not too extreme. ${ }^{39}$ There was homogeneity of variances, as assessed by Levene's test for equality of variances, $\mathrm{P}=0.473$. The interaction effect between framing and visual type was not statistically significant, $\mathrm{F}(1,335)=2.488, \mathrm{P}=0.116$, partial $\eta^{2}=0.007$. Therefore, an analysis of the main effect of both framing and visual type was performed, which indicated there was no statistically significant main effect of framing on intent to vaccinate, $\mathrm{F}(1,335)=1.761, \mathrm{P}=0.185$, partial $\eta^{2}=0.005$. In addition, there was no statistically significant main effect of visual type on intent to vaccinate, $\mathrm{F}(1,335)=0.125, \mathrm{P}=0.724$, partial $\eta^{2} \leq 0.001$. Therefore, the study's hypothesis was not supported. The research questions show there to be no difference between visual types, and no interaction between visual type and message framing on intent to get the future Zika vaccine.

\section{Intermediate outcomes}

A secondary aim of the current study was to determine what message characteristics are most effective at increasing intermediate psychosocial constructs that may contribute to intent to get the Zika vaccine. Again, two-way ANOVAs were conducted to address this aim. For these tests, data were largely normally distributed, as assessed by Q-Q plots. There were six outliers, as assessed as standardized scores being greater than 3.0. These outliers were left in the analysis. There was homogeneity of variances for all variables, as assessed by Levene's test for equality of variances with $p>05$. None of the interactions were significant. Therefore, an analysis of the main effect of both framing and visual type was performed for all intermediate outcomes. These results can be found in Table 1; the significant results are also outlined below.

\section{Main effects of framing}

First, a main effect of framing was present on subjective norms as a composite score consisting of five items: Gain-framed messages were associated with a higher subjective norm related to the Zika vaccine $\left(\mathrm{P}=0.005\right.$, partial $\left.\mathrm{h}^{2}=0.019\right)$. When assessing these items individually, a main effect of framing was present on subjective norm operationalized as people who are important to me would approve of me getting a future Zika vaccination $(\mathrm{P}=0.007$, partial $\left.\mathrm{h}^{2}=0.018\right)$; my family would approve of me getting a future Zika vaccination $\left(\mathrm{P}=0.003\right.$, partial $\left.\mathrm{h}^{2}=0.023\right)$; my friends would approve of me getting a future Zika vaccination ( $\mathrm{P}=0.002$, partial $\left.\mathrm{h}^{2}=0.024\right)$; my primary care provider would approve of me getting a future Zika vaccination $\left(\mathrm{P}=0.009\right.$, partial $\left.\mathrm{h}^{2}=0.017\right)$. Gainframed messages resulted in higher subjective norms item scores than loss-framed messages.

Second, a main effect of framing was present on perceived benefits as a composite score consisting of two items $(\mathrm{P}=0.016$, partial $\left.h^{2}=0.014\right)$. When considering the individual items, a main effect of framing was present on perceived benefits (of a future Zika vaccine), operationalized as $A$ future Zika vaccination will help me feel less worried about Zika $\left(\mathrm{P}=0.015\right.$, partial $\left.\mathrm{h}^{2}=0.014\right)$ and A future Zika vaccination will decrease my chance of getting Zika or its complications $\left(\mathrm{P}=0.038\right.$, partial $\left.\mathrm{h}^{2}=0.009\right)$.

Finally, a main effect of framing was present on self-efficacy as a composite score consisting of two items $(\mathrm{P}=0.032$, partial $\mathrm{h}^{2}=0.010$ ). When considering the individual items, a main effect of framing was present on self-efficacy operationalized as If I wanted to, I am confident I could get the future Zika vaccination $(\mathrm{P}=0.030$, partial $\left.\mathrm{h}^{2}=0.011\right)$. However, the other item How certain are you that you could get the future Zika vaccination, was not significant. 
Table 1. Two-way ANOVA results.

\begin{tabular}{|c|c|c|c|c|c|c|c|c|c|c|c|c|}
\hline \multirow[t]{2}{*}{ Variable } & \multicolumn{4}{|c|}{ Interaction } & \multicolumn{4}{|c|}{ Gain/Loss } & \multicolumn{4}{|c|}{ Photo/Infographic } \\
\hline & F & df & P & partial $\eta^{2}$ & F & df & $\mathbf{P}$ & partial $\eta^{2}$ & F & df & $\mathbf{P}$ & partial $\eta^{2}$ \\
\hline Attitude & 1.197 & 1,335 & 0.275 & 0.004 & 1.966 & 1,335 & 0.081 & 0.006 & 0.755 & 1,335 & 0.385 & 0.002 \\
\hline Subjective norms & 2.275 & 1,335 & 0.132 & 0.007 & 6.546 & 1,335 & $0.005^{*}$ & 0.019 & 0.065 & 1,335 & 0.799 & $<0.001$ \\
\hline Perceived Behavioral Control & 0.282 & 1,335 & 0.596 & 0.001 & 1.329 & 1,335 & 0.125 & 0.004 & 0.008 & 1,335 & 0.929 & 0.001 \\
\hline Perceived severity & 2.566 & 1,335 & 0.110 & 0.008 & 0.593 & 1,335 & 0.221 & 0.002 & 0.283 & 1,335 & 0.595 & 0.001 \\
\hline Perceived susceptibility & 0.036 & 1,335 & 0.850 & $<0.001$ & 1.642 & 1,335 & 0.101 & 0.005 & 3.065 & 1,335 & 0.081 & 0.009 \\
\hline Perceived benefits & 0.440 & 1,335 & 0.508 & 0.001 & 4.665 & 1,335 & $0.016^{*}$ & 0.014 & 0.046 & 1,335 & 0.829 & $<0.001$ \\
\hline Perceived barriers & 0.161 & 1,335 & 0.689 & $<0.001$ & 0.030 & 1,335 & 0.431 & $<0.001$ & 1.813 & 1,335 & 0.179 & 0.005 \\
\hline Perceived barriers incl. emotion & 0.615 & 1,335 & 0.433 & 0.002 & 0.460 & 1,335 & 0.249 & 0.001 & 0.013 & 1,335 & 0.908 & $<0.001$ \\
\hline Self-efficacy & 0.660 & 1,335 & 0.417 & 0.002 & 3.471 & 1,335 & $0.032 *$ & 0.010 & 1.687 & 1,335 & 0.195 & 0.005 \\
\hline Foolish-wise & 0.484 & 1,335 & 0.487 & 0.002 & 1.532 & 1,335 & 0.109 & 0.005 & 0.504 & 1,335 & 0.478 & 0.002 \\
\hline Harmful-beneficial & 1.019 & 1,335 & 0.314 & 0.003 & 4.333 & 1,335 & $0.019 *$ & 0.013 & 0.513 & 1,335 & 0.475 & 0.002 \\
\hline Worthless-Valuable & 1.615 & 1,335 & 0.205 & 0.005 & 1.753 & 1,335 & 0.093 & 0.005 & 0.957 & 1,335 & 0.329 & 0.003 \\
\hline Bad-good & 0.292 & 1,335 & 0.589 & 0.001 & 0.258 & 1,335 & 0.306 & 0.001 & 0.200 & 1,335 & 0.655 & 0.001 \\
\hline Negative-positive & 2.713 & 1,335 & 0.100 & 0.008 & 2.297 & 1,335 & 0.066 & 0.007 & 1.579 & 1,335 & 0.210 & 0.005 \\
\hline Zika vax out of own control & 1.204 & 1,335 & 0.273 & 0.004 & 0.892 & 1,335 & 0.173 & 0.003 & 0.318 & 1,335 & 0.573 & 0.001 \\
\hline Up to me to get Zika vax & 0.036 & 1,335 & 0.850 & $<0.001$ & 1.062 & 1,335 & 0.152 & 0.003 & 0.257 & 1,335 & 0.612 & 0.001 \\
\hline Zika vax: very little control & 0.059 & 1,335 & 0.807 & $<0.001$ & $<0.001$ & 1,335 & 0.496 & $<0.001$ & 0.880 & 1,335 & 0.349 & 0.003 \\
\hline Norms: people important to me & 3.389 & 1,335 & 0.067 & 0.010 & 6.065 & 1,335 & $0.007^{*}$ & 0.018 & 0.028 & 1,335 & 0.867 & $<0.001$ \\
\hline Norms: family would approve & 2.026 & 1,335 & 0.156 & 0.006 & 7.818 & 1,335 & $0.003^{*}$ & 0.023 & 0.164 & 1,335 & 0.686 & $<0.001$ \\
\hline Norms: friends would approve & 2.044 & 1,335 & 0.154 & 0.006 & 8.282 & 1,335 & $0.002^{*}$ & 0.024 & 0.018 & 1,335 & 0.894 & $<0.001$ \\
\hline Norms: PCP would approve & 3.335 & 1,335 & 0.069 & 0.010 & 5.719 & 1,335 & $0.009 *$ & 0.017 & 0.047 & 1,335 & 0.828 & $<0.001$ \\
\hline Norms: want to please people & 0.018 & 1,335 & 0.894 & $<0.001$ & 0.351 & 1,335 & 0.227 & 0.001 & 0.988 & 1,335 & 0.321 & 0.003 \\
\hline Benefits: less worry & 0.290 & 1,335 & 0.591 & 0.001 & 4.797 & 1,335 & $0.015^{*}$ & 0.014 & 0.187 & 1,335 & 0.665 & 0.001 \\
\hline Benefits: less chance at Zika & 0.465 & 1,335 & 0.496 & 0.001 & 3.178 & 1,335 & $0.038^{*}$ & 0.009 & 0.001 & 1,335 & 0.970 & $<0.001$ \\
\hline Barriers: interfere with activities & 0.572 & 1,335 & 0.450 & 0.002 & 0.099 & 1,335 & 0.377 & $<0.001$ & 0.043 & 1,335 & 0.835 & $<0.001$ \\
\hline Barriers: fear of needles & 0.435 & 1,335 & 0.510 & 0.001 & 0.078 & 1,335 & 0.390 & $<0.001$ & 2.301 & 1,335 & 0.130 & 0.007 \\
\hline Barriers: inconvenience & 0.316 & 1,335 & 0.574 & 0.001 & 0.340 & 1,335 & 0.280 & 0.001 & 2.732 & 1,335 & 0.099 & 0.008 \\
\hline Barriers: expensive & 0.416 & 1,335 & 0.519 & 0.001 & 0.199 & 1,335 & 0.328 & 0.001 & 2.969 & 1,335 & 0.086 & 0.009 \\
\hline Barriers: where to get vax & 0.003 & 1,335 & 0.959 & $<0.001$ & 0.016 & 1,335 & 0.450 & $<0.001$ & 0.569 & 1,335 & 0.451 & 0.002 \\
\hline Emotion vax: fear & 0.569 & 1,335 & 0.451 & 0.002 & 0.280 & 1,335 & 0.299 & 0.001 & 1.580 & 1,335 & 0.210 & .005 \\
\hline Emotion vax: nervous & 0.022 & 1,335 & 0.881 & $<0.001$ & 0.436 & 1,335 & 0.255 & 0.001 & 2.247 & 1,335 & 0.135 & 0.007 \\
\hline Emotion vax: confusion & 0.039 & 1,335 & 0.843 & $<0.001$ & 0.161 & 1,335 & 0.345 & $<0.001$ & 6.415 & 1,335 & $0.012^{*}$ & 0.019 \\
\hline Emotion vax: anger & 0.080 & 1,335 & 0.778 & $<0.001$ & 1.484 & 1,335 & 0.112 & 0.004 & 3.708 & 1,335 & 0.055 & 0.011 \\
\hline Emotion vax: cynicism & 0.046 & 1,335 & 0.831 & $<0.001$ & 0.009 & 1,335 & 0.463 & $<0.001$ & 0.724 & 1,335 & 0.396 & 0.002 \\
\hline Self-efficacy: confidence & 0.436 & 1,335 & 0.510 & 0.001 & 3.572 & 1,335 & $0.030^{*}$ & 0.011 & 0.277 & 1,335 & 0.599 & 0.001 \\
\hline Self-efficacy: certainty & 0.741 & 1,335 & 0.390 & 0.002 & 2.645 & 1,335 & 0.053 & 0.008 & 3.488 & 1,335 & 0.063 & 0.010 \\
\hline Susc: high chance at infection & 1.053 & 1,335 & 0.306 & 0.003 & 0.495 & 1,335 & 0.241 & 0.001 & 3.561 & 1,335 & 0.060 & 0.011 \\
\hline Susc: possibility of infection & 0.284 & 1,335 & 0.595 & 0.001 & 0.843 & 1,335 & 0.180 & 0.003 & 5.013 & 1,335 & $0.026^{*}$ & 0.015 \\
\hline Susc: worry about likelihood & 0.324 & 1,335 & 0.569 & 0.001 & 1.306 & 1,335 & 0.127 & 0.004 & 0.452 & 1,335 & 0.502 & 0.001 \\
\hline Susc: chance high mosquitoes & 0.180 & 1,335 & 0.672 & 0.001 & 2.080 & 1,335 & 0.075 & 0.006 & 1.240 & 1,335 & 0.266 & 0.004 \\
\hline Severity: serious complications & 0.065 & 1,335 & 0.799 & $<0.001$ & 1.421 & 1,335 & 0.117 & 0.004 & 1.360 & 1,335 & 0.244 & 0.004 \\
\hline Severity: very sick with Zika & 2.789 & 1,335 & 0.096 & 0.008 & 0.124 & 1,335 & 0.363 & $<0.001$ & 1.006 & 1,335 & 0.316 & 0.003 \\
\hline Severity: afraid of getting Zika & 2.319 & 1,335 & 0.129 & 0.007 & 0.033 & 1,335 & 0.428 & $<0.001$ & 0.048 & 1,335 & 0.826 & $<0.001$ \\
\hline Severity: complications pregnancy & 1.108 & 1,335 & 0.293 & 0.003 & 0.003 & 1,335 & 0.480 & $<0.001$ & 0.797 & 1,335 & 0.373 & 0.002 \\
\hline Severity: afraid to get pregnant & 0.946 & 1,335 & 0.332 & 0.003 & 0.916 & 1,335 & 0.170 & 0.003 & 0.757 & 1,335 & 0.385 & 0.002 \\
\hline Emotion virus: fear & 0.041 & 1,335 & 0.839 & $<0.001$ & 1.769 & 1,335 & 0.090 & 0.005 & 0.527 & 1,335 & 0.469 & 0.002 \\
\hline Emotion virus: nervous & 0.082 & 1,335 & 0.775 & $<0.001$ & 1.457 & 1,335 & 0.114 & 0.004 & 1.355 & 1,335 & 0.245 & 0.004 \\
\hline Emotion virus: confusion & 0.798 & 1,335 & 0.372 & 0.002 & 0.120 & 1,335 & 0.365 & $<0.001$ & 0.451 & 1,335 & 0.502 & 0.001 \\
\hline Emotion virus: anger & 0.294 & 1,335 & 0.588 & 0.001 & $<0.001$ & 1,335 & 0.498 & $<0.001$ & 0.262 & 1,335 & 0.609 & 0.001 \\
\hline Emotion virus: cynicism & 0.001 & 1,335 & 0.980 & $<0.001$ & 0.005 & 1,335 & 0.472 & $<0.001$ & 0.421 & 1,335 & 0.517 & 0.001 \\
\hline
\end{tabular}




\section{Main effects of visual type}

No main effect of visual type was present for any of the composite scores. Among individual items, only two individual items displayed a main effect of visual type: perceived barriers operationalized as the likelihood to feel confused about a future Zika vaccine, $(\mathrm{P}=0.012)$; the unweighted marginal means of confusion was $2.86 \pm 0.116$ for photo-based messages and $2.45 \pm 0.110$ for infographic-based messages. In addition, a main effect of visual type was present on perceived susceptibility operationalized as Getting infected with Zika is currently a possibility for me, $(\mathrm{P}=0.026)$; the unweighted marginal means of $3.33 \pm 0.127$ for infographic-based messages and $2.92 \pm 0.134$ for photo-based messages.

\section{Discussion}

This study examined the effect of Zika message framing (gain vs. loss) and image type (photo vs. infographic) on future Zika vaccine uptake intent and other psychosocial outcomes, using a $2 \times 2$ between-subjects experiment conducted via an online survey. The initial research question asked what message characteristics would be most effective at increasing intentions to get a future Zika vaccine, but the corresponding analyses yielded no significant results and the accompanying hypotheses were not supported. There are a number of plausible reasons for this outcome: First and foremost, the dose of the intervention - the frequency and length of exposure - may not have been strong enough with a single image exposure, and repeated exposure to the message may be needed, ${ }^{40}$ which is supported by research on encoded exposure in media content. ${ }^{41}$ This brief exposure, however, is typical for social media in general and Instagram in particular. Second, respondents may not have read the message or read it for comprehension. Third, the survey was carried out in early March, considered off-season for mosquitoes in much of the U.S. The public conversation about Zika at this time was less intense than during the previous summer, a time of heightened risk perception and widespread media coverage. Other factors that were not manipulated in the current study may have influenced the outcome include message source and virality. For example, whether the message was shared through a trusted Instagram connection (e.g., friend or relative) instead of directly from the CDC could potentially influence responses. Additionally, the study posts were portrayed with limited virality (i.e., the engagement frequency; 22 likes); increased virality may have increased the salience of and response to the post. Another consideration regarding the lack of differences in reported intent to get the Zika vaccine between gain- and loss-framed messages: Both gain- and loss-framed messages may be equally effective in promoting Zika vaccine messages when taking a population approach to prevention. Although this study recruited only women of reproductive age, there were very few women in the sample who were currently trying to conceive. It is possible that this high risk population may respond differently to messages focused on threats to a fetus than those women not currently trying to conceive. This could indicate the relevance of using both types of message frames for Zika vaccine public health communication campaigns.

The third research question asked what message characteristics are most effective at increasing intermediate psychosocial constructs that contribute to intent to get a future Zika vaccine. While no interaction effects were present, there were a few significant main effects. First, a main effect of framing was present for the subjective norm composite variable, as well as for four of the five subjective norm items - valuing the opinion people important to the person in general, parents, friends, and primary care. In spite of what the literature states about loss-framed messages being more effective in promoting vaccinations, the current study did not support this. Moreover, gain-framed messages were more effective in increasing subjective norms related to a future Zika vaccine uptake. In addition, gain-framed messages were also more effective in increasing perceived benefits of a future Zika vaccine, and, to a lesser extent, self-efficacy related to the vaccine. For example, gain-framed messages emphasized the benefits of getting the vaccine (e.g., it helps you stay healthy), while loss-framed messages underscored the consequences of not getting the vaccine (e.g., you will be at risk for having a baby with microcephaly).

This is a novel finding given that the existing literature indicates that loss-framed messages are more beneficial in promoting vaccine uptake and vaccine uptake intent. The unique nature of the future Zika vaccine could provide a potential explanation: While the vaccine is administered to women, the most salient prevention affects the (potential) fetus. O'Keefe and Nan suggest that people may be differentially susceptible to gain $v s$. loss-framed vaccine messages depending on whether the vaccine is for themselves or for their child(ren). ${ }^{42}$ In addition, even though the available literature shows indications that loss-framed messages are more effective than gain-framed messages when promoting vaccines, the debate over effectiveness of gain- $v s$. loss-framed messages related to vaccines is not settled. ${ }^{42} \mathrm{~A}$ final potential reason for the apparent effect of gain-framed messages in this study could be that, since the Zika vaccine is not available to the public yet, no reports about perceived adverse effects of the vaccine exist at this time. Thus, the vaccine may be perceived as less risky, which points to a greater effectiveness of gain-framed promotion messages on psychosocial predictors of intent. Future research should examine whether these findings are reproduced when the Zika vaccine becomes available.

\section{Strengths and limitations}

There are several limitations of the current study that should be taken into account in interpreting the findings. First, the exposure to the message was brief. Thus, the dose was likely insufficient to produce meaningful engagement with the content at the level needed to promote change in intent. Second, this study was carried out in March of 2017, when Zika was perceived as less of a threat than during the summer of 2016. However, it was an ethical consideration to not make the loss frame messages elicit too high of a threat in absence of a vaccine that would provide the necessary response efficacy to control the danger felt. In addition, the CDC was used as the source for the intervention messages. Results may be different based on the perceived origin of messages, such as a news source or a post from a trusted friend. Moreover, Puerto Rico, the U.S. territory with the highest prevalence of Zika thus far, was excluded from the experiment. Puerto Rico residents may well have had different responses to the intervention messages. Future studies should include Puerto Rico as well as include other nations affected by Zika. Finally, this experiment was implemented before a Zika vaccine was available to the general public. This means that the public had not experienced the benefits of the vaccine first hand. Conversely, perceived adverse effects of the vaccine were not present yet, either. Both factors could influence intent to get the vaccine. Therefore, it would be beneficial for this study to be repeated once the vaccine is available, and during the peak summer months when the perceived threat of Zika is higher. The intervention could then be adapted by increasing exposure to the messages. In addition, visuals consisting of moving images such as videos were outside the scope of this study, but should be considered for future studies. Moreover, the experimental messages primarily focused on the adverse effects of Zika on pregnant women and 
their fetuses. Other images are needed focused on both other Zika adverse effects and on other populations. In addition, messages are needed that emphasize threats to self versus potential offspring among women of reproductive age, as not all women in this age bracket are or intend to become pregnant.

\section{Conclusions and future directions}

The current visual, Instagram-targeted, social media intervention did not find an effect on reported future Zika vaccine uptake intent, and resulted in limited effects on intermediate outcomes that could lead to either future Zika vaccine uptake intent or future Zika vaccine uptake. This is likely indicative of the intervention not being dosed sufficiently for the desired result. This underscores the importance of not over-estimating the efficacy of social media messages as stand-alone interventions and emphasizes the importance of continued research into effective integration of these types of interventions as part of a larger campaign.

However, based on this study, it seems there might be an effect of gain-framed visual messaging on subjective norm and many of its items (the importance of parents', friends', and healthcare providers' opinions in the decision to get a future Zika vaccine). In addition, there may be a smaller effect of gain-framed messaging on the perceived benefits of a future Zika vaccination as well as self-efficacy related to the vaccine. Therefore, public health and health communication professionals should consider targeting social norms and perceived benefits related to the Zika vaccine, using gain-framed messages, especially when considering platforms like Instagram. Communication campaigns should include repeated exposure to messages to enhance dose and increase potential effects. Because social media is still a relatively recent phenomenon, and there still is a relative paucity of research into the field, it is essential to continue to expand the small but growing body of interdisciplinary research in this area.

Correspondence: Jeanine P.D. Guidry, Robertson School of Media and Culture, Virginia Commonwealth University, 901 W. Broad St. Suite 2216, Richmond, VA 23284, USA

Tel.: + 1.804.683.4512.

E-mail: guidryjd@vcu.edu

Key words: Zika, Zika vaccine, framing, visuals.

Contributions: JPDG, substantial contributions to the conception and design of the work; and well as the acquisition, analysis, and interpretation of data for the work; drafting the work for important intellectual content; and final approval of the version to be published. The co-authors substantially contributed to the design of the work and the analysis and interpretation of data for the work, revising it critically for important intellectual content, and final approval of the version to be published. This paper is derived from the dissertation of the first author, the other authors were the dissertation committee. Conflict of interest: the authors declare no potential conflict of interest. Funding: none.

Received for publication: 15 August 2017.

Revision received: 23 February 2018.

Accepted for publication: 13 April 2018.

(C) Copyright J.P.D. Guidry et al., 2018

Licensee PAGEPress, Italy

Journal of Public Health Research 2018;7:1162

doi:10.4081/jphr.2018.1162

This work is licensed under a Creative Commons Attribution NonCommercial 4.0 License (CC BY-NC 4.0).

\section{References}

1. World Health Organization. Situation report: Zika virus, microcephaly, Guillain-Barre Syndrome. 2017. Available from: http://apps.who.int/iris/bitstream/10665/254714/1/zikasitrep10Mar17-eng.pdf?ua=1.

2. Johansson MA, Mier-y-Teran-Romero L, Reefhuis J, et al. Zika and the risk of microcephaly. N Engl J Med 2016;375:14.

3. Lee ST, Basnyat I. From press release to news: mapping the framing of the 2009 H1N1 A influenza pandemic. Health Commun 2013;28:119-32.

4. Dredze M, Broniatowski DA, Hilyard KM. Zika vaccine misconceptions: a social media analysis. Vaccine 2016;34:3441-2.

5. Southwell BG, Dolina S, Jimenez-Magdaleno K, et al. Zika virus-related news coverage and online behavior, United States, Guatemala, and Brazil. Emerg Infect Dis 2016;22: 1320-1.

6. Constine J. Instagram's growth speeds up as it hits 700 million users. 2017. Available from: https://techcrunch.com/ 2017/ 04/26/instagram-700-million-users/.

7. Seltzer E, Horst-Martz E, Lu M, Merchant RM. Public sentiment and discourse about Zika virus on Instagram. Public Health 2017;150:170-5.

8. Gallagher KM, Updegraff JA. Health message framing effects on attitudes, intentions, and behavior: a meta-analytic review. Ann Behav Med 2012;43:101-16.

9. Rothman AJ, Bartels RD, Wlaschin J, Salovey P. The strategic use of gain $\square$ and loss $\square$ framed messages to promote healthy behavior: How theory can inform practice. J Commun 2006;56 S202-20.

10. Garcia-Retamero R, Cokely ET. Effective communication of risks to young adults: using message framing and visual aids to increase condom use and STD screening. J Exp Psychol Appl 2011;17:270.

11. Gerend MA, Shepherd JE, Monday KA. Behavioral frequency moderates the effects of message framing on HPV vaccine acceptability. Ann Behav Med 2008;35:221-9.

12. Abhyankar P, O'connor DB, Lawton R. The role of message framing in promoting MMR vaccination: Evidence of a lossframe advantage. Psychol Health Med 2008;13:1-16.

13. Nan X, Xie B, Madden K. Acceptability of the H1N1 vaccine among older adults: the interplay of message framing and perceived vaccine safety and efficacy. Health Commun 2012;27:559-68.

14. Briones RL, Nan X, Madden K, Waks L. When vaccines go viral: An analysis of HPV vaccine coverage on YouTube. Health Commun 2012;27:478-85.

15. Love B, Himelboim I, Holton A, Stewart K. Twitter as a source of vaccination information: Content drivers and what they are saying. Am J Infect Control 2013;41:568-70.

16. Guidry JPD, Carlyle K, Messner M, Jin Y. On pins and needles: How vaccines are portrayed on Pinterest. Vaccine 2015;33:5051-6.

17. Lipkus IM. Numeric, verbal, and visual formats of conveying health risks: suggested best practices and future recommendations. Med Decision Making 2007;27:696-713.

18. Ancker JS, Senathirajah Y, Kukafka R, Starren JB. Design features of graphs in health risk communication: A systematic review. J Am Med Inform Assoc 2006;13:608-18.

19. Houts PS, Doak CC, Doak LG, Loscalzo MJ. The role of pictures in improving health communication: a review of research on attention, comprehension, recall, and adherence. Patient Educ Couns 2006;61:173-90. 
20. McWhirter JE, Hoffman-Goetz L. A systematic review of visual image theory, assessment, and use in skin cancer and tanning research. J Health Commun 2014;19:738-57.

21. Paivio A. Dual coding theory: Retrospect and current status. Can J Psychol 1991;45:255.

22. Nielsen. 2016 Nielsen Social Media Report. 2017. Available from: http:/www.nielsen.com/us/en/insights/reports/2017/ 2016-nielsen-social-media-report.html.

23. Lazard A, Atkinson L. Putting environmental infographics center stage: The role of visuals at the Elaboration Likelihead Model's critical point of persuasion. Sci Commun 2015;37:633.

24. Occa A, Suggs LS. Communicating breast cancer screening with young women: an experimental test of didactic and narrative messages using video and infographics. J f Health Commun 2015;21:1-11.

25. Börzsei LK. Makes a meme instead: A concise history of internet memes. New Media Studies Magazine 2013.

26. Gerend MA, Shepherd JE. Using message framing to promote acceptance of the human papillomavirus vaccine. Health Psychol 2007;26:745.

27. Gerend MA, Shepherd JE. Predicting human papillomavirus vaccine uptake in young adult women: Comparing the health belief model and theory of planned behavior. Ann Behav Med 2012;44:171-80.

28. Ajzen I. The theory of planned behavior. Organizational behavior and human decision processes Psychol Health 2011;26:1113-27.

29. Rosenstock I. Historical origins of the Health Belief Model. Health Educ Monographs 1974;2:324-473.

30. Mo PKH, Lau JTF. Influenza vaccination uptake and associated factors among elderly population in Hong Kong: the application of the Health Belief Model. Health Educ Res 2015; 30:706-18.

31. Gargano LM, Painter JE, Sales JM, et al. Seasonal and 2009 H1N1 influenza vaccine uptake, predictors of vaccination, and self-reported barriers to vaccination among secondary school teachers and staff. Hum Vaccines 2011;7:89-95.
32. Chen MF, Wang RH, Schneider JK, et al., Using the health belief model to understand caregiver factors influencing childhood influenza vaccinations. J Commun Health Nurs 2011; 28:29-40.

33. Myers LB, Goodwin R. Determinants of adults' intention to vaccinate against pandemic swine flu. BMC Public Health 2011;11.

34. Brewer NT, Fazekas KI. Predictors of HPV vaccine acceptability: a theory-informed, systematic review. Prev Med 2007;45:107-14.

35. Bennett KK, Buchanan JA, Adams AD. Social-cognitive predictors of intention to vaccinate against the human papillomavirus in college-age women. J Soc Psychol 2012;152:48092.

36. Chew C, Eysenbach G. Pandemics in the age of twitter: content analysis of tweets during the 2009 H1N1 outbreak. PLoS One 2010;5.

37. Fu KW, Liang H, Saroha N, et al. How people react to Zika virus outbreaks on Twitter? A computational content analysis. Am J Infect Control 2016;44:1700-2.

38. Simmons JP, Nelson LD, Simonsohn U. False-positive psychology: Undisclosed flexibility in data collection and analysis allows presenting anything as significant. Psychol Sci 2011; 22:1359-66.

39. Cohen J, Cohen P, West SG, Aiken LS. Applied multiple regression/correlation analysis for the behavioral sciences. Outliers and multicollinearity: diagnosing and solving regression problem II. London: Taylor \& Francis; 2003. p. 390-430.

40. Farrelly MC, Davis KC, Haviland ML, et al. Evidence of a dose-response relationship between truth antismoking Ads and youth smoking prevalence. Am J Public Health 2005;95:42531.

41. Southwell BG. Between messages and people: A multilevel model of memory for television content. Commun Res 2005;32:112-40.

42. O'Keefe DJ, Nan X. The relative persuasiveness of gain-and loss-framed messages for promoting vaccination: A meta-analytic review. Health Commun 2012;27:776-83. 